\title{
Fragments of fullerenes: novel syntheses, structures and reactions
}

\author{
Lawrence T. Scott \\ Department of Chemistry, Merkert Chemistry Center \\ Boston College, Chestnut Hill, Massachusetts 02167-3860 USA
}

\begin{abstract}
The utility of high-temperature gas-phase cyclization reactions for constructing bowl-shaped polycyclic aromatic hydrocarbons related to $\mathrm{C}_{60}$ was first demonstrated by our simple synthesis of corannulene, 1. This strategy has subsequently been extended to the preparation of larger fullerene fragments and promises to play a central role in future syntheses of even more spectacular molecular bowls, baskets and tubes. Isomerization reactions of benzenoid hydrocarbons at high temperatures in the gas phase have also been studied and are now known to occur by reversible benzene ring contractions to fulvene intermediates. The relationship of these small molecule rearrangements to the mechanisms of fullerene production and isomerization ("annealing") is discussed. Finally, the conversion of simple benzenoid hydrocarbons to fragments of fullerenes containing fivemembered rings with two hydrogens fewer is shown to be a common high-temperature reaction of bay region polycyclic aromatic compounds, and a preliminary mechanistic proposal is offered along with supporting experimental evidence.
\end{abstract}

The landmark synthesis of corannulene, 1, by Barth and Lawton was first announced in 1966 (1), more than two decades prior to the earliest preparative synthesis of $\mathrm{C}_{60}$ and the higher fullerenes (2). This marvelous bowl-shaped $\mathrm{C}_{20} \mathrm{H}_{10}$ hydrocarbon represents the minimal structural subunit of a fullerene that retains a curved network of trigonal carbon atoms, and recent years have witnessed a resurgence of interest in corannulene and the larger fragments of fullerenes $(3,4)$. My coworkers and I have been actively engaged in this area of research since 1990 , and the first section of this paper presents an overview of our syntheses and selected transformations of bowl-shaped polycyclic aromatic hydrocarbons (PAHs). The latter portion of the paper describes some of our findings on the high temperature chemistry of PAHs that we believe provide insight into the mechanism of formation and isomerization of fullerenes.

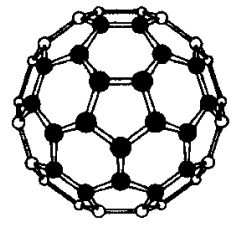

Buckminsterfullerene $C_{60}$

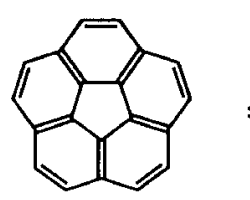

Corannulene 1

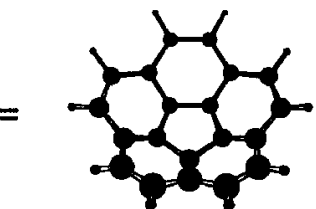

$$
\text { (n) }
$$$$
\text { . }
$$

\section{Strategies for Constructing Bowl-Shaped Fragments of Fullerenes}

How can one fashion a curved network of trigonal carbon atoms when all the constituent atoms have a natural tendency to remain planar? Any rational synthesis of a bowl-shaped fullerene fragment must overcome the angle strain associated with pyramidalization of numerous $\mathrm{sp}^{2}$ hybridized carbon atoms. Barth and Lawton solved this problem by first putting together the entire carbon skeleton of corannulene with very few centers of unsaturation and then aromatizing the rings in the final step $(1,5)$. Our approach, on the other hand, is completely different. Inspired by the recognition that fullerenes, the most strikingly curved networks of trigonal carbon atoms, can be formed under high energy conditions in the gas phase $(2,6)$, we reasoned that it should be possible to capitalize on the principles at work there for a new synthesis of corannulene and related fragments of fullerenes. Toward this end, we prepared 7,10-diethynylfluoranthene, 2 , and subjected it to flash pyrolysis (FVP) at $1000-1100^{\circ} \mathrm{C}$. To our delight, corannulene was formed as the sole isolable product (7). Scheme 1 depicts the mechanism we envision to be operating in this reaction.

Scheme 1
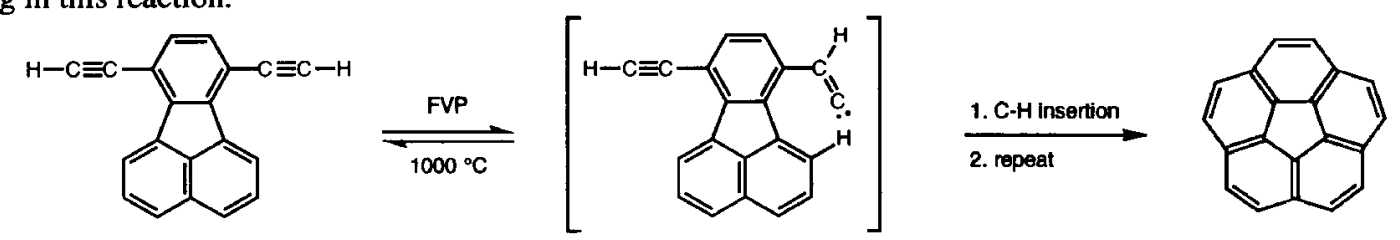
From the early work of R. F. C. Brown et al. we knew that terminal alkynes could be equilibrated with the isomeric vinylidenes by flash vacuum pyrolysis at temperatures above $650^{\circ} \mathrm{C}$ and that carbenes generated reversibly in this manner could be trapped intramolecularly (8). For a two-carbon side chain to reach across the fluoranthene bay region, however, the ring system had to be coaxed temporarily into an abnormal conformation, and we relied on the high energy conditions of the gas phase pyrolysis to accomplish that. The idea worked, and we were on our way to adding some new chapters to the corannulene story.

An improved synthesis that we now employ to prepare gram quantities of corannulene in just three steps from commercially available starting materials is summarized in Scheme $2(9,10)$. The initial assembly of a disubstituted fluoranthene takes place in a single reaction vessel without isolation of intermediates, and the final pyrolysis induces the thermal loss of $\mathrm{HCl}$ from both side chains to generate our earlier diethynylfluoranthene, 2 , which cyclizes as before. To our knowledge, this was the first time that 1-chlorovinyl groups had been used as "masked acetylenes" in a pyrolysis.

Scheme 2

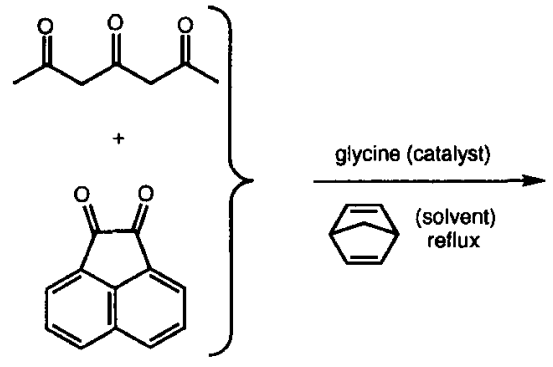

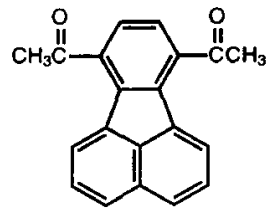

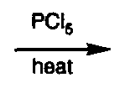

first step via:<smiles>CC(=O)C1=C(C(C)=O)c2cccc3cccc1c23</smiles>

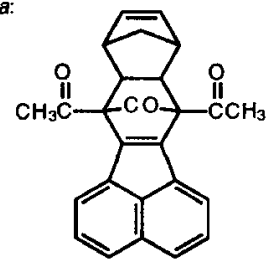

The intramolecular trapping of aryl radicals represents an attractive alternative to carbene cyclizations, as illustrated by our synthesis of dibenzo[a,g]corannulene, 3 , in Scheme $3(11,12)$. Thermal cleavage of carbon-bromine bonds can be induced at temperatures below $1000^{\circ} \mathrm{C}$, but the second cyclization requires pyrolysis at $1000-1100^{\circ} \mathrm{C}$.

Scheme 3
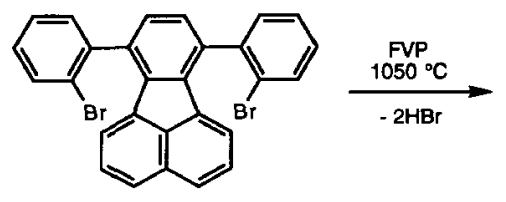

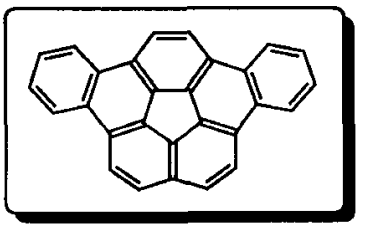

dibenzo[a,g]corannulene 3

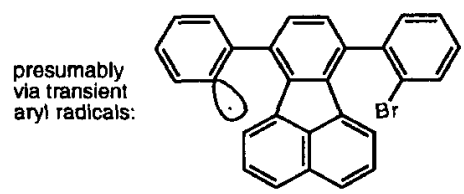

We have recently applied this same strategy to a synthesis of the alkene-centered fullerene fragment, 4 (Scheme 4) (13). Extensions to syntheses of larger bowl-shaped PAHs are currently under investigation.

\section{Scheme 4}

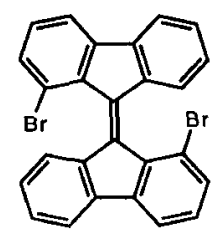

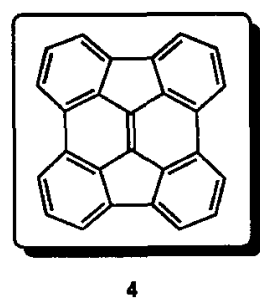

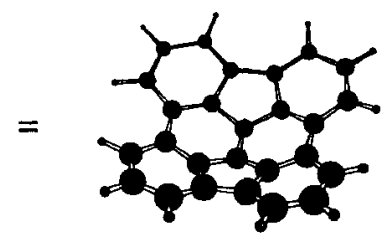

$A C_{96} H_{12}$ Bowl

As an obvious alternative (and complement) to starting from precursors that already contain all the carbon atoms of the final target molecule, it seemed to us that one should be able to start from a corannulene base and build deeper molecular bowls and baskets by successive annulation reactions. A simple demonstration of this strategy is illustrated in Scheme $5(14,15)$.

Scheme 5

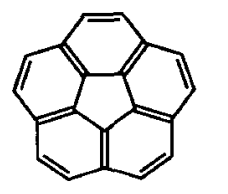

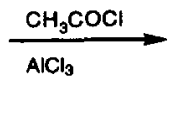

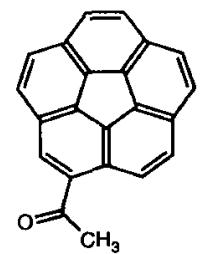

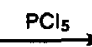

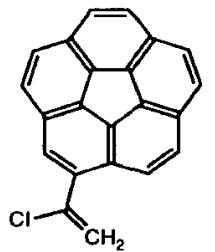

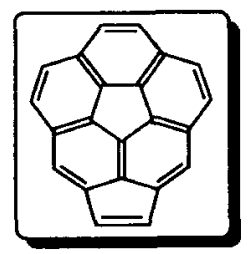

(c) 1996 IUPAC, Pure and Applied Chemistry 68, 291-300 
A more powerful approach, of course, would be to bridge several notches around the rim in a single operation. Once we found that Friedel-Crafts alkylation of corannulene with t-butyl chloride gives the beautifully symmetrical penta-t-butyl derivative, 5 , as the major product (16), it did not take long to find conditions to prepare the corresponding pentachloro derivative, 6 (Scheme 6) (16).

Scheme 6
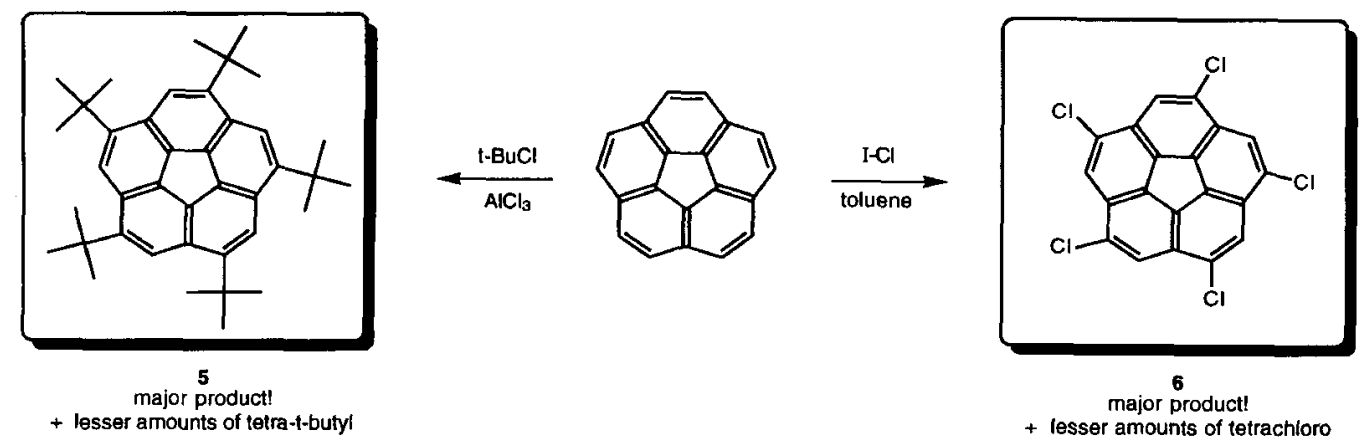

+ lesser amounts of tetra-t-butyl

Nickel-catalyzed coupling of 6 with trimethylsilylethynyl magnesium bromide has subsequently yielded pentaalkyne 7 (Scheme 7) (16), which we are now examining as a possible precursor to the $\mathrm{C}_{30} \mathrm{H}_{10}$ hemifullerene, 8 .

Scheme 7<smiles>Clc1cc2c(Cl)cc3c(Cl)cc4c(Cl)cc5c(Cl)cc1c1c5c4c3c21</smiles>

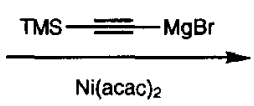

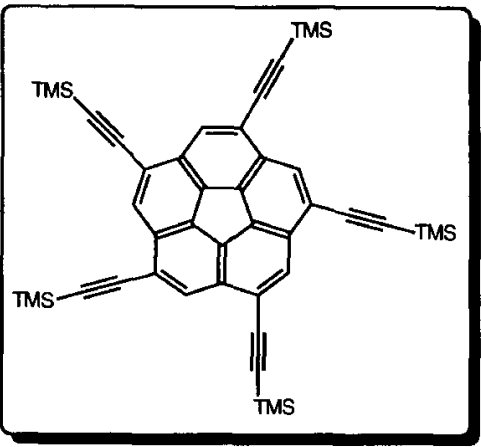

7

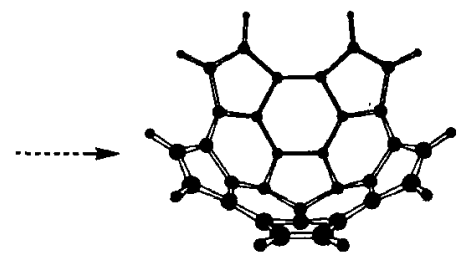

Target $=\mathrm{C}_{30} \mathrm{H}_{10}$

8

More vigorous chlorination of corannulene gives good yields of the decachloro derivative, 9 (11), which we have used to prepare a family of donor-substituted corannulenes, e.g., 10 (Scheme 8) (16). Preliminary experiments have been initiated to extend the coupling reaction in Scheme 7 to a synthesis of decaethynylcorannulene, an attractive $\mathrm{C}_{40} \mathrm{H}_{10}$ bowl precursor.

\section{Scheme 8}
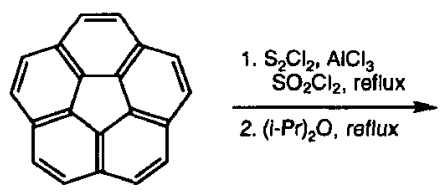

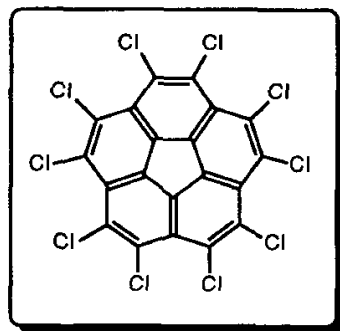

9

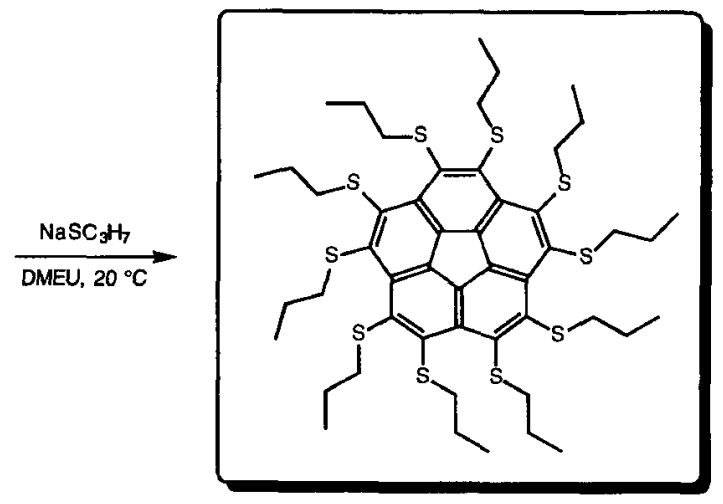

10

Good progress has been made in the development of useful strategies for the synthesis of bowl-shaped fullerene fragments, and many examples of high-temperature gas phase syntheses have begun to appear $(3,4)$, but we believe the most exciting opportunities still lie ahead.

\section{The Thermal Benzene Ring Contraction Rearrangement}

Concurrent with the synthetic work described above, we have continued our research on the thermal benzene ring contraction rearrangement (Scheme 9), a fundamental reaction of benzenoid hydrocarbons that was discovered in our laboratories nearly twenty years ago (17) and has recently been invoked as a key element in proposals to explain the mechanisms of formation and isomerization of fullerenes. 
The 1,2-Switch of Carbon Atoms in Benzenoid Hydrocarbons. We first encountered this remarkable rearrangement during our studies on the thermal automerization of naphthalene (17). In a flow pyrolysis at temperatures in the $1000-1100{ }^{\circ} \mathrm{C}$ range, $\left[1-{ }^{13} \mathrm{C}\right]$ naphthalene suffers reversible ring contraction to benzofulvene, which results in scrambling of the isotopic label between positions 1 and 2 (Scheme 10); none of the label ever leaks into the angular position of the naphthalene, even after several half lives of scrambling. We have good experimental evidence $(18,19)$ that this ring contraction is not concerted but is actually a stepwise process in which the hydrogen migration precedes the carbon-carbon bond shift, as predicted by theoretical calculations (20).

Scheme 9

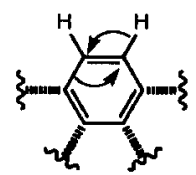

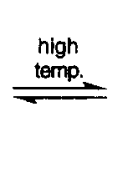

Scheme 10

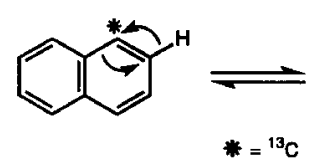<smiles>C=C=C</smiles><smiles>[C+]1=CC=Cc2ccccc21</smiles>

Over the years, we have found the 1,2-switch of carbon atoms to be practically a universal reaction of benzenoid hydrocarbons. Scheme 11 pictures a collection of ${ }^{13} \mathrm{C}$-labeled benzenoid hydrocarbons that we have studied (17, $21,22,23)$; the arrows indicate the two adjacent positions in each compound between which the label becomes scrambled at high temperatures. In none of these cases does the label ever migrate to any position in the molecule other than the one adjacent to the original site of the label, i.e., the scrambling is always a 1,2-switch. We have even observed the thermal rearrangement of [ortho- ${ }^{13} \mathrm{C}_{2}$ ]benzene to $\left[\right.$ meta $-{ }^{13} \mathrm{C}_{2}$ ]benzene at $1100{ }^{\circ} \mathrm{C}$ and, to a lesser extent, secondary rearrangement of the [meta- $\left.{ }^{13} \mathrm{C}_{2}\right]$ benzene to $\left[\right.$ para- $\left.{ }^{13} \mathrm{C}_{2}\right]$ benzene (24). Such rearrangements must be going on all the time wherever benzenoid hydrocarbons are subjected to high temperatures, such as during combustion, coal liquefaction, and petroleum hydrotreating. Without the isotopic labels, however, they are invisible!

\section{Scheme 11}<smiles>c1ccc2c3c(ccc2c1)CC3</smiles><smiles>C1=CC2CC1=Cc1cc3ccccc3cc12</smiles><smiles>c1ccc2c(c1)ccc1ccc3c(c12)CC3</smiles><smiles></smiles><smiles></smiles><smiles>c1ccc2c(c1)C1CCC2c2c1ccc1ccccc21</smiles><smiles></smiles>

The Transposition of 5- and 6-Membered Rings. The loss of aromaticity and the introduction of angle strain clearly make benzene ring contractions highly endothermic, so it is not surprising that fulvenes fail to accumulate in the above pyrolyses. It occurred to us, however, that we might be able to arrest the naphthalene automerization (Scheme 10) at the benzofulvene stage if we were to incorporate an etheno bridge across the 1-and 8-positions, i.e., start with acenaphthylene. In such a molecule, contraction of a benzene ring from a six-membered ring to a fivemembered ring would be accompanied by the simultaneous ring expansion of the original five-membered ring to a new benzene ring, and the overall rearrangement would be degenerate, thereby rendering it thermoneutral. This hope was realized by the ${ }^{13} \mathrm{C}$-labeling experiments summarized in Scheme 12.

By synthesis, we prepared both compound 12 and compound 15 . We then subjected them individually to flash pyrolysis at $c a .1000^{\circ} \mathrm{C}$. As predicted, compound 12 gives isomers 11 and 13 in equal amounts (under conditions of low conversion) by benzene ring contractions involving the shifts of bonds $\mathbf{a}$ and $\mathbf{b}$, respectively (25). In contrast to the naphthalene automerization (Scheme 10), there is no energetic driving force for these products of benzene ring contraction rearrangements to re-expand the newly formed five-membered rings, so the primary rearrangement products are now observable. When this rearrangement is carried out to higher conversion, the secondary rearrangement product, 14, begins to appear among the products at the expense of 13, again in agreement with expectations (25). Finally, pyrolysis of compound 15 gives isomers 14 and 16 in equal amounts (under conditions of low conversion) by benzene ring contractions involving the shifts of bonds a and b, respectively (19).

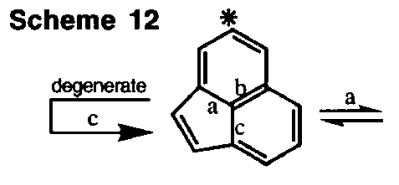

11

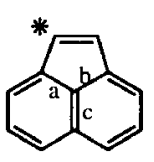

12

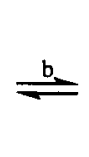

1

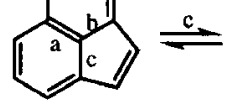

13

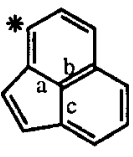

14
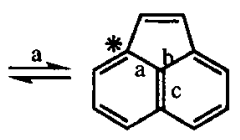

15

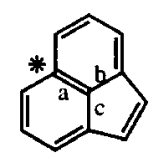

16

It bears noting that the benzene ring contraction rearrangements in Scheme 12 are completely equivalent to those that lead to the 1,2-switch of carbon atoms in simple benzenoid hydrocarbons, despite the superficial difference that the isotopic label in acenaphthylene appears to jump from one ring of the molecule to another with each rearrangement, i.e., never a 1,2-switch of carbon atoms. In fact, the eleven-atom rim of the acenaphthylene remains intact throughout this scrambling while the three spokes emanating from the twelfth carbon atom at the hub simply migrate around in succession.

Conceptually, one can view this process as the migration of a five-membered ring from one position in the tricyclic network to another, and it is from this perspective that an appreciation of the thermal benzene ring contraction rearrangement can help to demystify the mechanisms of formation and isomerization of fullerenes. Under the high- 
energy conditions employed for fullerene production, five-membered rings should be able to "migrate" in this manner throughout the polycyclic network of trigonal carbon atoms until the most stable fullerene is reached. The notion of a thermodynamically driven "annealing" was introduce very early in the fullerene literature (26) but without a mechanistic picture of the sort presented here.

A special case of the benzene ring contraction rearrangement commonly referred to as the "Stone-Wales rearrangement" (27) has been much discussed in recent years as a potential mechanism for fullerene annealing (28), and we have devoted some effort to searching for an authentic example of such a process in a small molecule.

The "Stone-Wales Rearrangement." Scheme 13 depicts the bonding changes that define a "Stone-Wales rearrangement" for a generic fullerene; if all the other rings are preserved in their initial orientations, such a process would produce a new isomer of the original fullerene $(27,28)$.

Scheme 13
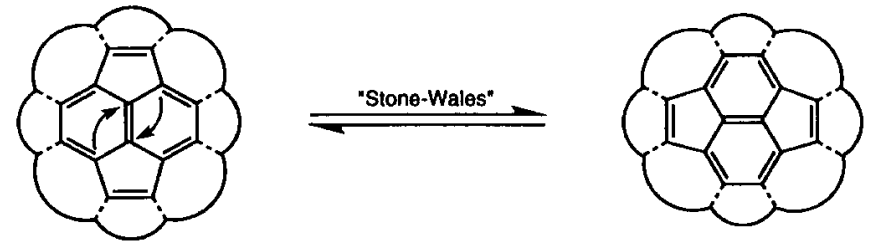

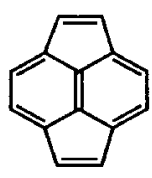

pyracylene 17

Using thermal cyclization reactions akin to those in Scheme 1, we have developed a new synthesis of pyracylene, 17, the smallest fullerene fragment in which this rearrangement might occur (29). By design, our methods permitted us to label all the acetylenic carbon atoms as ${ }^{13} \mathrm{C}$-isotopes. When the cyclization reaction was performed with labeled precursor, however, the pyracylene was found to be labeled exclusively in the five-membered rings, i.e., no rearrangement had scrambled any of the label into the six-membered rings (Scheme 14). This was true even when the cyclization (itself an exothermic process) was carried out at $1100^{\circ} \mathrm{C}$.

Scheme 14<smiles>[C]#Cc1cccc2c(C#C[CH])cccc12</smiles>

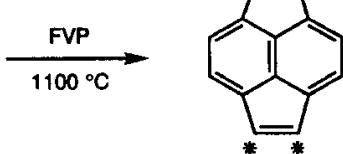

isolated

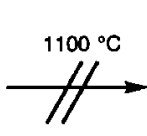

nono seen by NMR

From our experiments, therefore, it is quite clear that the parent pyracylene molecule, 17, does NOT rearrange according to the Stone-Wales pathway at $1100^{\circ} \mathrm{C}$. In fact, there is to this day not a single example of a bona fide "Stone-Wales rearrangement" of a polycyclic aromatic hydrocarbon that we know of. Does this mean that such processes are not involved in the annealing and isomerization of fullerenes? Not necessarily; we believe the "StoneWales rearrangement" is still a plausible process on the surface of fullerenes for the reasons outlined in the following section.

$\pi$ Bond Order Correlation. From the accumulation of all our experience with the thermal benzene ring contractions and related rearrangements that lead to the 1,2-switch of carbon atoms (including some not mentioned above), we have noted a qualitative correlation between the ease of rearrangement and the "double bond character" at the site of the rearrangement $(17,21,22,23,24,30)$. Figure 1 shows a full range of compounds, from terminal acetylenes, which scramble their carbon atoms at temperatures as low as $550^{\circ} \mathrm{C}(31)$, to pyracylene, which fails to rearrange even at $1100^{\circ} \mathrm{C}$ under pyrolysis conditions that are identical except for the temperature. The calculated Coulson $\pi$ bond orders at the site of rearrangement for these compounds (given in parentheses) decrease monotonically in the order shown, and so does the ease of rearrangement.
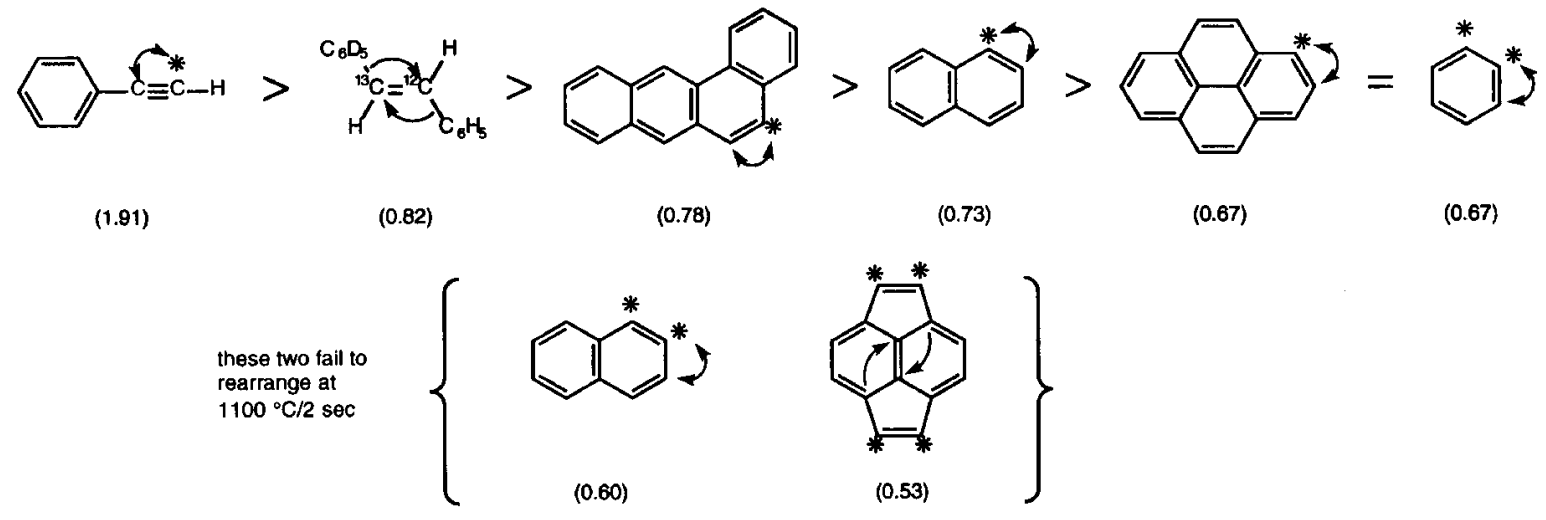

Fig. 1. Correlation between the ease of thermal 1,2-shift rearrangement and the $\pi$ bond order (Coulson $p_{\mathrm{ij}}$ values shown in parentheses) at the site of the rearrangement. 
Thus, it is the exceptionally low $\pi$ bond order between the two central carbon atoms of pyracylene that accounts for the recalcitrance of this molecule toward thermal benzene ring contraction. We hasten to point out, however, that the $\pi$ bond order between the corresponding atoms on $\mathrm{C}_{60}$ is significantly higher than that in pyracylene (27, 32). Furthermore, the strain energy associated with the curvature of a fullerene might cause a lowering of the activation energy for the "Stone-Wales rearrangement" relative to that in a planar molecule. Consequently, we do not consider our experiments to militate against the "Stone-Wales rearrangement" of fullerenes; the parent pyracylene molecule is simply a poor model for the corresponding ring system imbedded on a fullerene surface.

\section{Thermal Transformations of Bay Region Polycyclic Benzenoid Hydrocarbons into Fragments of Fullerenes}

During the course of our ${ }^{13} \mathrm{C}$-labeling studies on PAHs, we discovered that pyrolysis of chrysene, 18 , at $1100{ }^{\circ} \mathrm{C}$ leads to the formation of benzopyracylene (cyclopenta[cd]fluoranthene), 19 (Fig. 2) (23). At this temperature, the percent conversion is far from complete $(20-25 \%)$; however, the only other monomeric material obtained in any significant amount was the recovered chrysene (75-80\%). Thus, the transformation is a rather specific one, producing a single new compound. Unlike the thermal benzene rings contractions discussed above, however, the formation of 19 from chrysene is not an isomerization, since the product contains two hydrogen atoms fewer than the starting material.

We were instantly captivated by this transformation, because benzopyracylene, 19 , is a pentacyclic fragment of all known fullerenes, and its formation from chrysene looks just like the sort of process that must be occurring during the production of fullerenes from PAHs in flames (33). The unusual features of the transformation can be summarized as:

- a loss of two hydrogens to increase the $\mathrm{C} / \mathrm{H}$ ratio

- the permanent destruction of a benzene ring

- the creation of five-membered rings from a purely benzenoid compound

- an increase in the total number of rings

- the generation of a fullerene fragment

The Ubiquity of Such Processes. In the beginning, we had no clues regarding the mechanism of this extraordinary transformation, but it did seem reasonable to assume that the benzo group in 19 must have originated from one of the terminal rings in 18. Based on that assumption, we postulated that an analogous reaction of the chrysene homolog with an additional "spectator ring" on the other end, i.e., picene, 20, should lead to dibenzopyracylene (4,5-o-phenylenefluoranthene), 21. Furthermore, since benzannulation is known to lower the aromaticity of an adjacent ring system, we had reason to hope that picene might suffer the same reaction more<smiles>c1ccc2c(c1)ccc1ccccc12</smiles>

18<smiles>c1ccc2ccccc2c1</smiles>

22

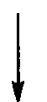

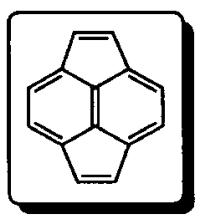

17

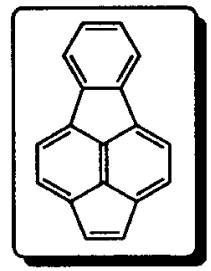

19<smiles>C1=Cc2ccccc2C=C2C=Cc3ccccc3C=C12</smiles>

23<smiles>c1cc2c3cccc(c3-2)c2cccc3cccc(c1)c32</smiles>

25<smiles>c1ccc2c(c1)ccc1c2ccc2c3ccccc3ccc21</smiles>

20<smiles>c1cc2cccc3c4cccc5ccc6cccc(c(c1)c23)c4c65</smiles>

24

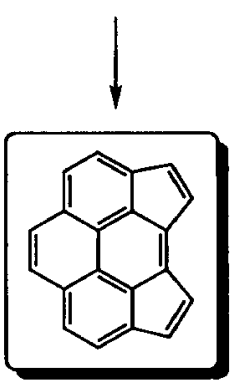

26

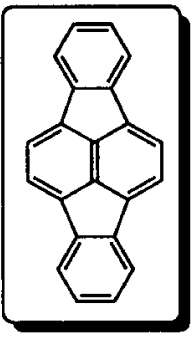

21

27

Fig. 2. Thermal transformations of bay region PAHs at $1100^{\circ} \mathrm{C}$. 
readily than chrysene. In fact, pyrolysis of picene at $1100^{\circ} \mathrm{C}$ gives a $50: 50$ mixture of 21 and recovered picene (Fig. 2) (23).

Removal of both "spectator ring" from picene leaves phenanthrene, 22, and we were pleased to see that even this robust PAH, with no extra rings to help lower the aromaticity of the central core, gives tiny amounts (ca. 5\%) of the corresponding product, pyracylene, 17 , upon pyrolysis at $1100^{\circ} \mathrm{C}$ (Fig. 2) (23).

Since the common structural feature of these PAHs seemed to be the presence of a bay region, we examined several other PAHs with bay regions and found them all to exhibit similar chemistry at $1100^{\circ} \mathrm{C}$. More specifically, benzopyracylene, 19, is generated by pyrolysis of benz[a]anthracene, 23 , and dicyclopenta[cd,fg]pyrene, 26 , is formed from compounds 24, 25, and 27 (Fig. 2) (23). None of these transformations proceeds with a percent conversion any higher than $50 \%$, but each gives essentially a single monomeric product. All involve the lose of two hydrogen atoms, except for $\mathbf{2 7} \rightarrow \mathbf{2 6}$, in which the elements of acetylene are lost. The deviant behavior of 27 in the last example, in fact, turned out to be our first mechanistic clue, and we will return to this point following the next section.

Independent Syntheses of the Resulting Dicyclopenta-PAHs. At the time when this work was performed, pyracylene, 17, and dibenzopyracylene, 21, were both known, but benzopyracylene, 19, was not, nor were any of the dicyclopentapyrenes, e.g., 26. To confirm the structural assignments we had made on the basis of spectroscopic evidence, therefore, we devised unambiguous syntheses of these new compounds. Scheme 15 outlines our synthesis of $19(23,34)$; it bears a close resemblance to our synthesis of pyracylene in Scheme 14 . A noteworthy feature of this synthesis is the preparation of a 1-chlorovinyl group directly from a trimethylsilylprotected acetylene.

Scheme 15
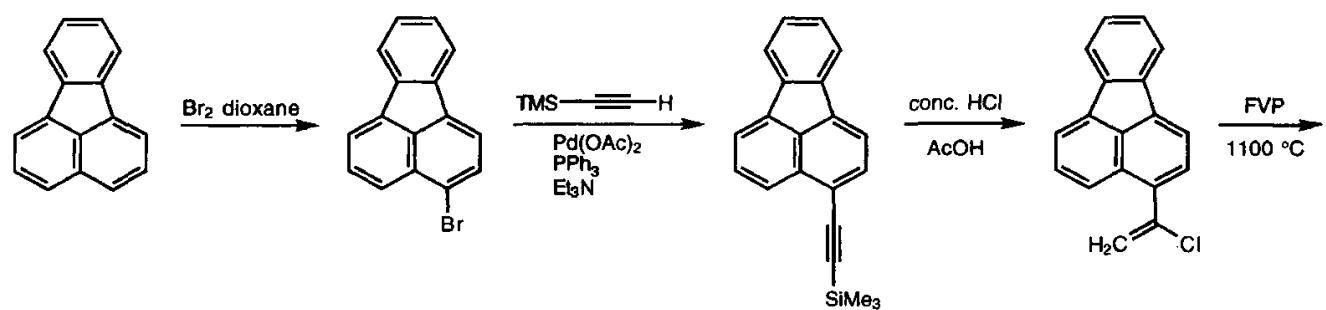
All three dicyclopentapyrenes were synthesized using analogous ring closure reactions (Scheme 16$)(34,35)$. In
these cases, the 1 -chlorovinyl groups were prepared by the action of $\mathrm{PCl}_{5}$ on the corresponding diacetylpyrenes.

Scheme 16<smiles></smiles><smiles>CC(=O)c1ccc2ccc3ccc(C(C)=O)c4ccc1c2c34</smiles><smiles>CC(=O)c1cc(C(C)=O)c2ccc3cccc4ccc1c2c43</smiles><smiles>CCCCCC(C)(C)C</smiles><smiles>C=C(Cl)c1ccc2ccc3c(C(=C)Cl)ccc4ccc1c2c43</smiles>

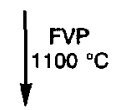<smiles>c1cc2c3ccc4cc5ccc6ccc7cc(ccc1CC2)c7c6c5c3-4</smiles><smiles>C=C(Cl)c1ccc2ccc3ccc(C(=C)Cl)c4ccc1c2c34</smiles>
$\stackrel{\text { FVP }}{1100^{\circ} \mathrm{C}}$

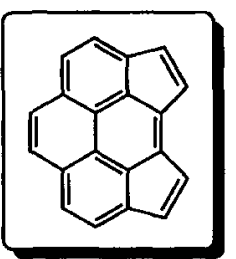

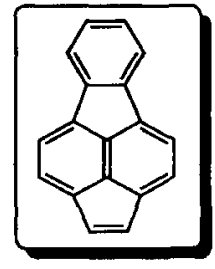

19

\section{n}


A Unified Mechanistic Picture. The surprising loss of acetylene from compound 27, followed by conversion to 26, the same product as that derived from perylene, 24, spawned the idea that these two reactions might be proceeding by way of the common intermediate 28 . From this admittedly speculative hypothesis, we extrapolated to consider the possibility that bay region diradicals of the same sort might be responsible for all the transformations in Fig. 2. Such a proposal seemed amenable to experimental test, since it predicts that the same products should be formed by independent generation of the proposed intermediates. We therefore focused our attention on the system that gave the worst conversion, i.e., phenanthrene to pyracylene $(22 \rightarrow 17, c a$. 5\% conversion). The bay region diradical intermediate in this case would be 29 . Ketone $\mathbf{3 0}$ constituted an attractive precursor to this intermediate, and we were delighted to find that pyracylene, 17, is not only formed from 30 at $1100{ }^{\circ} \mathrm{C}$, but it is formed in substantially greater amounts than from phenanthrene, 22 (23). This conversion of 30 to 17 certainly makes bay region diradicals appear plausible as intermediates in the bay region PAH transformations summarized in Fig. 2; however, we hardly consider this proof against the involvement of alternate pathways. Further experiments along these lines are currently in progress.

How could such diradicals isomerize to the observed dicyclopenta-PAHs? One attractive possibility involves coupling of the radical centers, followed by rearrangement steps as illustrated in Scheme 17.

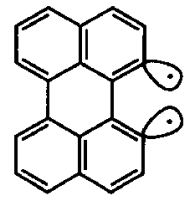

28

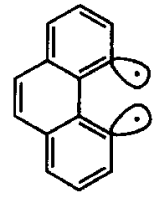

29

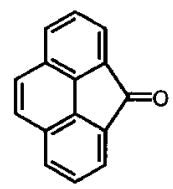

30

Scheme 17

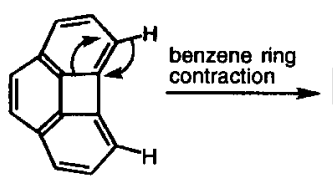

31

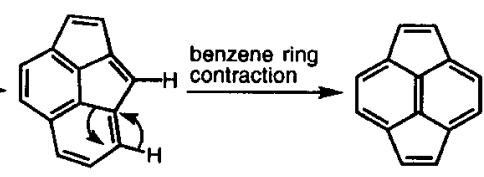

32

17

The proposed four-membered ring intermediate, 31, though highly strained and calculated to adopt a bowl-shaped geometry, must certainly lie lower in energy than diradical 29 , so its formation seems plausible. The final transposition of 5- and 6-membered rings from 32 to 17, on the other hand, simply represents a special case of the acenaphthylene bond-switch rearrangement already discussed (cf. Scheme 12). It is interesting to note, with respect to fullerene chemistry, that the $32 \longrightarrow 17$ transformation can be viewed as an "annealing mechanism" by which this tetracyclic $\mathrm{C}_{14} \mathrm{H}_{8}$ system can satisfy the "isolated pentagon rule" $(36,37,38)$; calculations at the AM1 level of theory attach an exothermicity of $20 \mathrm{kcal} / \mathrm{mol}$ to this last step. The transformation of 4 - and 6-membered rings from 31 to 32 finds precedent in the thermal rearrangement of biphenylene, 33, to benzopentalene, 34, (Scheme 18) reported by Wiersum and Brown $(39,40)$. These authors have actually suggested a more circuitous mechanistic pathway from 33 to 34 , but the evidence is equally compatible with the one-step isomerization in Scheme 18.

\section{Scheme 18}

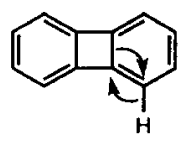

33

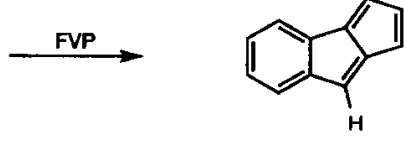

34

More problematical are the reports by Brown et al. $(41,42)$ in which our proposed intermediate 32 was previously generated by two other pathways and was shown to ring open to the two isomeric ethynylacenaphthylenes 36 and 37 (Scheme 19). We therefore repeated Brown's experiment to see if pyracylene, 17, might have been overlooked among the products. Indeed, we found that pyrolysis of 35 at $1100^{\circ} \mathrm{C}$ gives not only the two ethynylacenaphthylenes, 36 and 37, reported by Brown et al. but also pyracylene, 17, and, to a lesser extent, even a third ethynylacenaphthylene $(23,43)$. Furthermore, we saw the same three ethynylacenaphthylenes formed as products along with pyracylene in the pyrolysis of ketone 30. Thus, we consider intermediates $\mathbf{3 1}$ and $\mathbf{3 2}$ and their higher benzologs to be viable candidates to explain all the thermal transformations of bay region PAHs summarized in Fig. 2. The thermal conversion of triphenylene to cyclopent[hi]acephenanthrylene reported by Wiersum $(44,45)$ is likewise readily explained by this mechanism.

Scheme 19<smiles>C#Cc1ccc2cccc(C#C)c2c1</smiles>

35
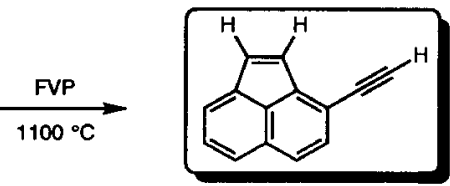

36

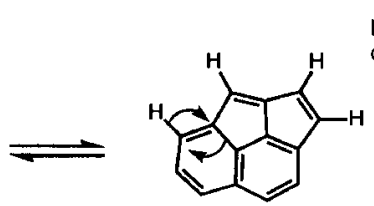

32

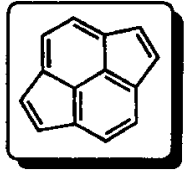

17

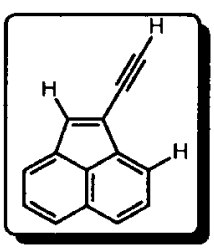

37 
As a direct test of our mechanistic proposal, we have recently conducted the ${ }^{13} \mathrm{C}$-labeling experiment summarized in Scheme 20 (23). In agreement with our prediction, the two isotopic labels originating in the central ring of picene end up exclusively at the indicated sites in the dibenzopyracylene resulting from thermolysis.

Scheme 20
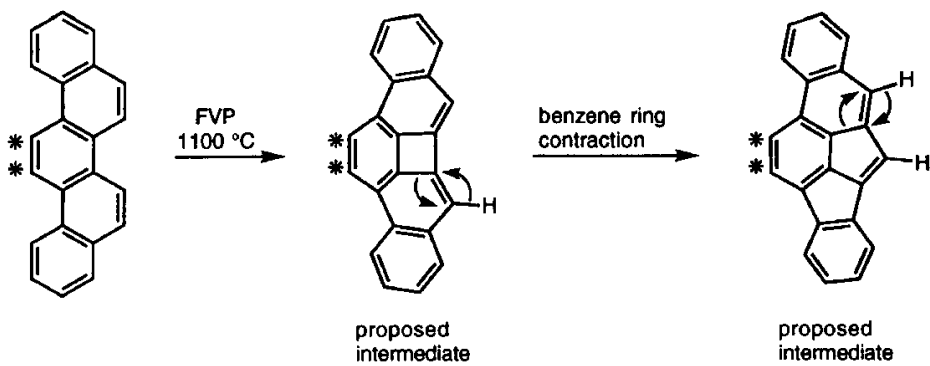

benzene ring

proposed

intermediate

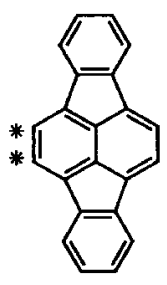

product obtained

\section{Future Prospects}

As the unconventional synthetic methods described here become more widely adopted, we can expect to see larger molecular bowls, baskets, belts, nanotubes and other polycyclic carbon networks related to fullerenes synthesized in the laboratory. So far, corannulene is the only such compound whose physical and chemical properties have been explored in even the most preliminary way, and the balance among "inside," "outside," and "edge" chemistry will be fascinating to explore as a function of molecular curvature.

The high temperature benzene ring contraction rearrangement is now reasonable well understood, and the role it plays in the mechanism of fullerene formation and isomerization will undoubtedly grow clearer as that picture continues to unfold. Our more recent discovery that a wide range of bay region PAHs suffer thermal transformations leading to the pairwise generation of five-membered rings, on the other hand, has posed new questions that are just beginning to be answered. Future mechanistic proposals to explain the production of fullerenes from hydrocarbon precursors in flames will almost certainly incorporate such reactions as pivotal steps. Whether bay region PAHs lose hydrogen directly to make bay region diradicals, e.g., 28 and 29, or they find their way by some other sequence of steps to four-membered ring intermediates, e.g., 31, and/or annulated pentalenes, e.g., 32, we will try to decide on the basis of future experiments.

\section{Acknowledgments}

My deepest appreciation goes to the many students and other collaborators whose names are cited in the references and without whom none of this work would have been done. Financial support from the Department of Energy, the National Science Foundation and the Petroleum Research Fund, administered by the American Chemical Society, is also gratefully acknowledged.

\section{References}

1. W. E. Barth, R. G. Lawton, J. Am. Chem. Soc. 88, 380-1 (1966).

2. W. Krätschmer, L. D. Lamb, K. Fostiropoulos, D. R. Huffman, Nature 347, 354-8 (1990).

3. J. S. Siegel, T. J. Seiders, Chem. in Britain, 313-6 (1995).

4. R. Faust, Angew. Chem. Internat. Ed. Engl. 34, 1429-32 (1995).

5. R. G. Lawton, W. E. Barth, J. Amer. Chem. Soc. 93, 1730-45 (1971).

6. H. W. Kroto, J. R. Heath, S. C. O'Brien, R. F. Curl, R. E. Smalley, Nature 318, 162-3 (1985).

7. L. T. Scott, M. M. Hashemi, D. T. Meyer, H. B. Warren, J. Am. Chem. Soc. 113, 7082-4 (1991).

8. R. F. C. Brown, F. W. Eastwood, G. P. Jackman, Aust. J. Chem. 30, 1757-67 (1977).

9. L. T. Scott, P.-C. Cheng, M. S. Bratcher, Seventh International Symposium on Novel Aromatic Compounds, abstr. no. 64, Victoria, British Columbia, Canada, July 19-24, 1992.

10. P.-C. Cheng, M.S. thesis, University of Nevada, Reno (1992).

11. L. T. Scott, P.-C. Cheng, M. S. Bratcher, E. Corpuz, unpublished results presented at the Twelfth IUPAC Conference on Physical Organic Chemistry, abstr. no. 34, Padova, Italy, August 28 - September 2, 1994.

12. L. T. Scott, M. S. Bratcher, unpublished results presented at the Symposium on Synthetic Hydrocarbon Chemistry, Loker Hydrocarbon Research Institute, University of Southern California, Los Angeles, December 1994.

13. L. T. Scott, N. Choi, recent results not previously published.

14. L. T. Scott, P.-C. Cheng, recent results not previously published. For a completely different synthesis of cyclopenta[bc]corannulene, starting from a preformed five-membered ring, see ref. 15 .

15. A. H. Abdourazak, A. Sygula, P. W. Rabideau, J. Am. Chem. Soc. 115, 3010-11 (1993).

16. L. T. Scott, P.-C. Cheng, recent results not previously published.

17. L. T. Scott, G. K. Agopian, J. Am. Chem. Soc. 99, 4506-7 (1977). 
18. L. T. Scott, M. M. Hashemi, T. H. Schultz, M. B. Wallace, J. Am. Chem. Soc. 113, 9692-3 (1991).

19. L. T. Scott, A. Racoveanu-Schiketanz, recent results not previously published.

20. K. M. Merz, Jr., L. T. Scott, J. Chem. Soc., Chem. Commun., 412-14 (1993).

21. L. T. Scott, M. A. Kirms, A. Berg, P. E. Hansen, Tetrahedron Lett. 23, 1859-62 (1982).

22. L. T. Scott, T. H. Tsang, L. A. Levy, Tetrahedron Lett. 25, 1661-4 (1984).

23. L. T. Scott, A. Necula, recent results not previously published.

24. L. T. Scott, N. H. Roelofs, T. H. Tsang, J. Am. Chem. Soc. 109, 5456-61 (1987).

25. L. T. Scott, N. H. Roelofs, Tetrahedron Lett. 29, 6857-60 (1988).

26. J. R. Heath, S. C. O'Brien, R. F. Curl, H. W. Kroto, R. E. Smalley, Comments Cond. Mater. Phys. 13, 119 (1987).

27. A. J. Stone, D. J. Wales, Chem. Phys. Lett. 128, 501-3 (1986).

28. J. M. Hawkins, M. Nambu, A. Meyer, J. Am. Chem. Soc. 116, 7642-5 (1994) and references cited therein.

29. L. T. Scott, H. B. Warren, Seventh International Symposium on Novel Aromatic Compounds, abstr. no. 65, Victoria, British Columbia, Canada, July 19-24, 1992.

30. L. T. Scott, N. H. Roelofs, K. Heisler, experimental results not previously published.

31. R. F. C. Brown, K. J. Harrington, G. L. McMullen, J. Chem. Soc., Chem. Commun., 123-4 (1974).

32. M. Ozaki, A. Takahashi, Chem. Phys. Lett. 127, 242-4 (1986).

33. J. B. Howard, et al., Carbon 30, 1183-201 (1992).

34. L. W. Jenneskens, Eighth International Symposium on Novel Aromatic Compounds, abstr. no. 7, Braunschweig, Germany, July 30-August 4, 1995 described closely related synthetic work; we thank Prof. Jenneskens for exchanging manuscripts prior to publication.

35. L. T. Scott, A. Necula, J. Org. Chem. 61, in press (1996).

36. T. G. Schmalz, W. A. Seitz, D. J. Klein, G. E. Hite, Chem. Phys. Lett. 130, 203 (1986).

37. H. W. Kroto, Nature 329, 529 (1987).

38. F. Diederich, et al., Science 254, 1768-70 (1991).

39. U. E. Wiersum, L. W. Jenneskens, Tetrahedron Lett. 34, 6615-18 (1993).

40. R. F. C. Brown, et al., Tetrahedron Lett. 35, 4405-8 (1994).

41. M. Adeney, R. F. C. Brown, K. J. Coulston, F. W. Eastwood, I. W. James, Aust. J. Chem. 44, 967-80 (1991).

42. R. F. C. Brown, F. W. Eastwood, N. R. Wong, Tetrahedron Lett. 34, 1223-6 (1993).

43. R. F. C. Brown, personal communication: reexamination of his original spectra reveals that pyracylene was indeed present in his pyrolysis samples but had simply been overlooked.

44. R. G. Neilen, U. E. Wiersum, Eighth International Symposium on Novel Aromatic Compounds, abstr. no. 107, Victoria, British Columbia, Canada, July 19-24, 1992.

45. R. G. Neilen, U. E. Wiersum, Janssen Chimica Acta 10, 3 (1992). 